\title{
Feasibility of evaluating the stenosis of intracranial segment in the vertebral artery using neck-brain integrated ultrasound: a comparison with computed tomography angiography and digital subtraction angiography
}

\author{
Jianjun Wang ${ }^{1}$, Qianfeng $\mathrm{Ma}^{1}$, Zhenxing Yang ${ }^{2}$, Liyuan $\mathrm{Ma}^{1}$ \\ ${ }^{1}$ Department of Ultrasonography, General Hospital of Ningxia Medical University, Yinchuan, China; ${ }^{2}$ Department of Neurology, General Hospital \\ of Ningxia Medical University, Yinchuan, China \\ Contributions: (I) Conception and design: J Wang; (II) Administrative support: J Wang, Q Ma; (III) Provision of study materials or patients: J Wang, Z \\ Yang, L Ma; (IV) Collection and assembly of data: All authors; (V) Data analysis and interpretation: J Wang, Q Ma, Z Yang; (VI) Manuscript writing: \\ All authors; (VII) Final approval of manuscript: All authors. \\ Correspondence to: Jianjun Wang. Department of Ultrasonography, General Hospital of Ningxia Medical University, 804 South Shengli Street, \\ Xingqing District, Yinchuan, China. Email: nxwang2018@163.com.
}

Background: Currently, no clear diagnostic indicator of vertebral artery hypoplasia (VAH) or intracranial stenosis exists in clinic. This study aims to study the feasibility of neck-brain integrated ultrasound for evaluating stenosis of the intracranial segment in the vertebral artery by comparing with those of computed tomography angiography (CTA) and digital subtraction angiography (DSA).

Methods: Clinical data of 138 patients who presented to our hospital with dizziness and headache as the main symptoms between April 2018 and June 2019 were retrospectively analyzed. Patients were divided into observation group and unilateral VAH group. The diagnosing accuracy of ultrasound, CTA, and DAS, the vertebral artery diameter (VAD), blood flow, end diastolic velocity (EDV), peak systolic velocity (PSV) values, resistance indexes (RIs), and difference of RI were compared between the two groups. The sensitivity, specificity and accuracy of ultrasound hemodynamic indicators in assessing stenosis of the intracranial segment of the vertebral artery were calculated.

Results: The accuracy rates of mild stenosis and non-stenosis with neck-brain integrated ultrasound were significantly different from those with CTA and DSA examination $(\mathrm{P}<0.05)$. The VAD, blood flow, EDV and PSV values of the affected side in the observation group were lower than those of the VAH side in the unilateral VAH group, while the RI and RI difference value were significantly higher than the unilateral VAH group $(\mathrm{P}<0.05)$. When the predicted boundary value was $2.084 \mathrm{~mm}(\mathrm{VAD}), 56.41 \mathrm{~mL} / \mathrm{min}$ (blood flow), $8.47 \mathrm{~cm} / \mathrm{s}$ (EDV), 0.743 (RI), and 0.149 (difference of RI), the sensitivity and specificity were $(86.43 \%$, 49.45\%), (88.67\%, 47.42\%), (80.47\%, 68.71\%), (82.71\%, 84.66\%), and (95.84\%, 80.11\%), respectively.

Conclusions: Neck-brain integrated ultrasound is of great value as a noninvasive examination in the diagnosis of stenotic lesions of the internal cervical vertebral artery. VAD, EDV, blood flow, RI, and the difference in RI can be used as indicators to evaluate stenosis of the internal carotid segment of the vertebral artery and provide a reliable basis for the clinical diagnosis of posterior circulation ischemia.

Keywords: Neck-brain integrated ultrasound; stenosis of intracranial segment in vertebral artery; computed tomography angiography (CTA); digital subtraction angiography (DSA)

Submitted Jul 08, 2020. Accepted for publication Sep 03, 2020.

doi: $10.21037 /$ atm-20-5713

View this article at: http://dx.doi.org/10.21037/atm-20-5713 


\section{Introduction}

Posterior circulation ischemic stroke, a clinical syndrome of vertebrobasilar artery (VBA) system ischemia, results from in situ thrombosis, embolism, or vascular stenosis $(1,2)$. It principally involves the brain tissue supplied by the VBA system (posterior circulation), including the brain stem, cerebellum, and part of the temporal lobe. Accounting for approximately $20-25 \%$ of all ischemic strokes, posterior circulation ischemic stroke carries a mortality rate of approximately $30 \%$ (3), and is mainly caused by vertebral artery stenosis or hypoplasia. Patients with a vertebral artery diameter $(\mathrm{VAD})$ of $\leq 2.5 \mathrm{~mm}$ are evaluated as simple vertebral artery hypoplasia (VAH) or coexisting intracranial stenosis. However, no clear diagnostic indicator exists for these conditions $(4,5)$. Therefore, this study retrospectively explored the feasibility of using integrated neck-brain integrated ultrasound to assess vertebral artery intracranial stenosis, and compared the diagnostic efficacy of this method with those of CT angiography (CTA) and digital angiography (DSA), to provide clinically appropriate diagnostic indicators. We present the following article in accordance with the STROBE reporting checklist (available at http://dx.doi.org/10.21037/atm-20-5713).

\section{Methods}

\section{General patient information}

The clinical data of 138 patients who presented at our hospital with dizziness and headache as the main symptoms from April, 2018, to June, 2019, were retrospectively analyzed. The patients were divided into two groups according to whether they had stenosis of the unilateral vertebral artery: the observation group and the unilateral VAH group. The observation group comprised 94 cases ( 51 males and 43 females), aged between $34-75$ years, with an average age of $41.36 \pm 10.64$ years. The unilateral VAH group comprised 44 cases (20 males and 24 females), aged between 33-76 years, with an average age of $42.52 \pm$ 11.37 years. There were no significant differences in the general data in the two groups $(\mathrm{P}>0.05)$.

The inclusion criteria were as follows: (I) the patient underwent integrated neck-brain integrated ultrasound, CTA, DSA, and surgery in our hospital; (II) complete imaging data; (III) no other serious malignant diseases; and (IV) no past surgical history. The exclusion criteria were as follows: (I) malignant diseases of other organs; (II) arteritis or moyamoya disease; (III) pregnant or lactating; (IV) non- vascular dizziness and headaches such as brain tumors; or (V) abnormal heart rhythm.

The grouping criteria were as follows. The observation group: a VAD of $\leq 2.5 \mathrm{~mm}$ on one side or a ratio of VAD to the contralateral side of $<1 / 1.7$, combined with dizziness, limbs disturbance, dysphagia, hemianopia, and other clinical symptoms, as well as different degrees of hypertension and dyslipidemia (6). The unilateral VAH group: a VAD of $\leq 2.5 \mathrm{~mm}$ on one side or a ratio of VAD to the contralateral side of $<1 / 1.7$, with no obvious stenosis of the vertebral artery, but with symptoms of posterior circulation ischemia. This study was approved by General Hospital of Ningxia Medical University (2020-586). All procedures performed in this study involving human participants were in accordance with the Declaration of Helsinki (as revised in 2013). Because of the retrospective nature of the research, the requirement for informed consent was waived.

\section{Examination methods}

The inspection instruments, PHILIPS EPIQ5 color Doppler ultrasound diagnostic apparatus, PHILIPS iCT 256-row spiral CT machine, and PHILIPSXPER FD20 DSA were all from Philips (Eindhoven, Netherlands).

A 3-12 MHz linear array ultrasound probe was used to routinely detect the intima-media thicknesses of the bilateral common carotid artery and the vertebral artery, and also the detection of the location, number and nature of plaque, degree of stenosis, and the blood flow resistance index and other indexes. To calculate the differences between the blood flow of the vertebral artery and resistance indexes of the two groups, the VAD, peak systolic velocity (PSV), end diastolic velocity (EDV), resistance index (PSV-EDV)/PSV, and mean time-averaged peak velocity (TAPV) were measured in systole. Vertebral artery blood flow $(\mathrm{mL} / \mathrm{min})=\pi \times(\mathrm{D} / 2)^{2} \times \mathrm{TAPV}(\mathrm{D}$ represented for $\mathrm{VAD}$ ), and the difference in the resistance index was the difference in the resistance index between the two vessels of the same subject. All parameters were tested for 3 times by experienced vascular sonographers and their mean values were taken.

CTA was administrated using the volume scanning mode, and the number of scanning layers was determined according to the extent of the lesion. First, a flat scan of the brain was performed. Following that, a high-pressure syringe was used to inject $80 \mathrm{~mL}$ of iohexol reagent. After the contrast medium was eliminated, the CTA scan of head and neck was performed, after which a $3 \mathrm{D}$ reconstruction 


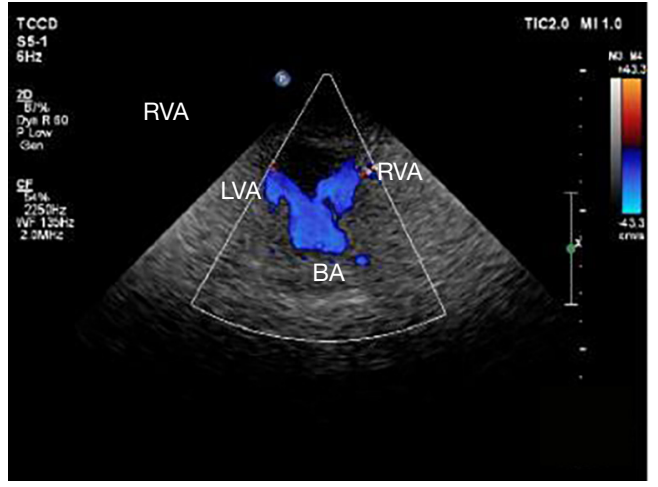

Figure 1 A neck-brain integrated ultrasound image showing vascular stenosis with abundant blood flow signals. LVA, left vertebral artery; RVA, right vertebral artery; BA, basilar artery.

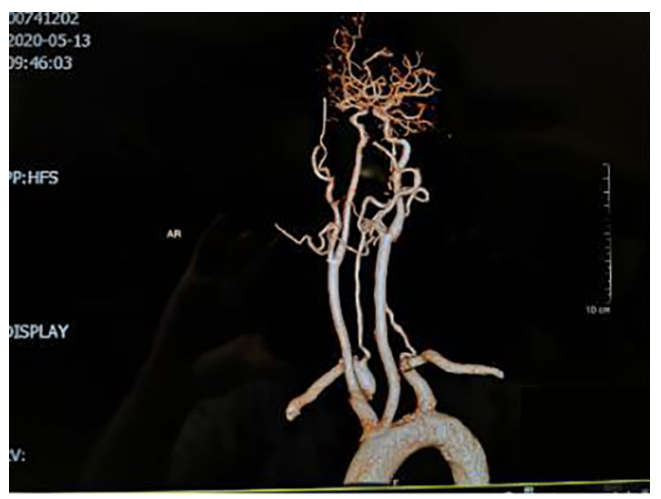

Figure 2 A computed tomography angiography (CTA) image showing mild narrowing of the vessels.

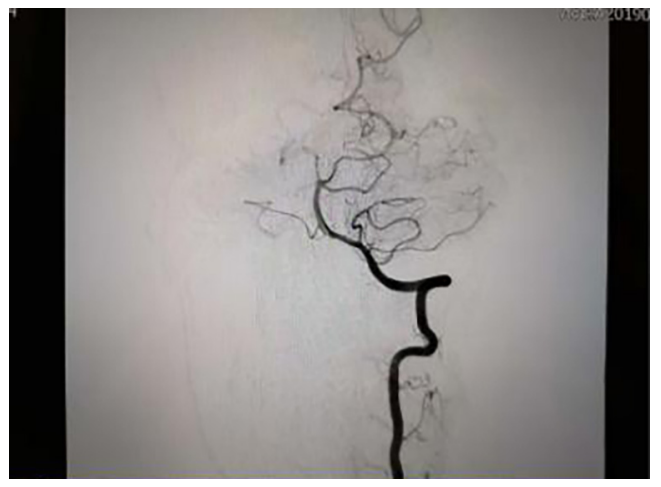

Figure 3 A digital subtraction angiography (DSA) image showing the vessels with mild stenosis. was performed. The DAS examination was carried out to magnify the angiography of stenotic lesions, followed by routine examination in the lateral and double oblique positions.

\section{Observation indexes}

The imaging results were reviewed by two or more experienced radiologists in the diagnostic team to reach a consensus. In case of disagreement, consensus can be reached through consultation. The accuracy of ultrasound, CTA, and DAS in diagnosing vascular plaque and stenotic lesions was compared. NASCET was used to study and analyze the degree of stenosis. Stenosis rate $=(1-\mathrm{a} / \mathrm{b})$ $\times 100 \%$, in which "a" is the stenosis lumen diameter and "b" is the normal carotid lumen diameter distal to the stenosis. The VAD, blood flow, EDV, and PSV were compared between the observation and the unilateral VAH groups. The RI and the difference in RI between the two groups were compared. The sensitivity, specificity, and accuracy of using ultrasound hemodynamic indicators to evaluate vertebral artery intracranial stenosis were analyzed.

\section{Statistical analysis}

All data were analyzed using SPSS 18.0 software (IBM, USA). Measurement data were described as mean \pm standard deviation, and analyzed using Student's $t$-test. Count data were expressed as rate or composition ratio, and analyzed using the $\chi^{2}$ test. $\mathrm{P}<0.05$ was considered to represent statistical significance.

\section{Results}

\section{Comparison of the examination accuracy of vascular stenosis in observation group using different diagnosis}

Neck-brain integrated ultrasound detected 101 vascular stenotic lesions in 94 patients (Figure 1), CTA detected 110 vascular lesions (Figure 2), and DSA detected 93 vascular lesions (Figure 3). The accuracy rates of mild stenosis and non-stenosis were significantly different with neck-brain integrated ultrasound, CTA, and DSA $(\mathrm{P}<0.05)$; however, there were no significant differences between the three examination techniques in the diagnosis of severe stenosis 
Table 1 Comparison of the accuracy of different examination methods in the diagnosis of vascular stenosis in the observation group (branch)

\begin{tabular}{lcccc}
\hline Examination method & Non-stenosis & Mild stenosis & Moderate to severe stenosis & Occlusion \\
\hline Neck-brain integrated ultrasound & 111 & 63 & 32 & 3 \\
CTA & 102 & 69 & 52 & 34 \\
DSA & 119 & 52 & 7 \\
\hline
\end{tabular}

CTA, computed tomography angiography; DSA, digital subtraction angiography.

Table 2 Comparison of VAD, blood flow, EDV, and PSV values between the observation group and the unilateral VAH group

\begin{tabular}{|c|c|c|c|c|c|c|c|c|}
\hline Groups & \multicolumn{2}{|c|}{ VAD (mm) } & \multicolumn{2}{|c|}{ Blood flow } & \multicolumn{2}{|c|}{$\mathrm{EDV}(\mathrm{cm} / \mathrm{s})$} & \multicolumn{2}{|c|}{$\mathrm{PSV}(\mathrm{cm} / \mathrm{s})$} \\
\hline $\begin{array}{l}\text { Observation } \\
\text { group }(n=94)\end{array}$ & $2.13 \pm 0.41$ & $3.81 \pm 0.67$ & $39.67 \pm 11.37$ & $382.14 \pm 77.65$ & $8.34 \pm 3.36$ & $20.59 \pm 4.71$ & $42.16 \pm 10.51$ & $55.36 \pm 10.43$ \\
\hline $\begin{array}{l}\text { Unilateral VAH } \\
\text { group }(n=44)\end{array}$ & $2.46 \pm 0.21$ & $4.01 \pm 0.53$ & $143.24 \pm 47.61$ & $401.64 \pm 77.84$ & $12.67 \pm 3.07$ & $20.94 \pm 4.36$ & $53.13 \pm 9.24$ & $57.62 \pm 11.41$ \\
\hline$P$ & 0.001 & 0.084 & 0.001 & 0.172 & 0.001 & 0.678 & 0.001 & 0.252 \\
\hline
\end{tabular}

$\mathrm{X}$ represents the affected side and the $\mathrm{VAH}$ side of the observation group and the unilateral VAH group, respectively; $\mathrm{Y}$ corresponds to the healthy side of the observation group and the unilateral VAH group, respectively. VAD, vertebral artery diameter; EDV, end diastolic velocity; PSV, peak systolic velocity; VAH, vertebral artery hypoplasia.

Table 3 Comparison of the RIs between the observation group and the unilateral VAH group

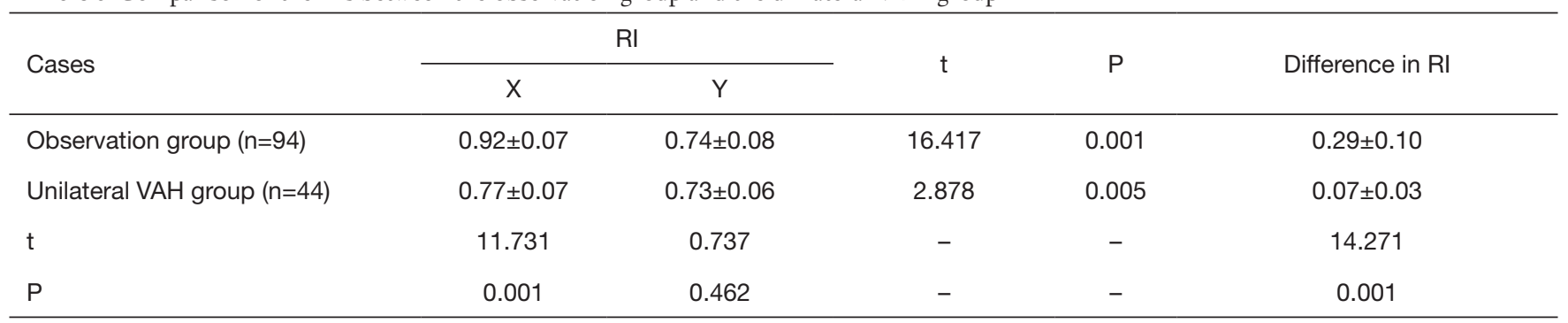

RI, resistance index; VAH, vertebral artery hypoplasia.

and occlusion $(\mathrm{P}>0.05$, Table 1$)$.

\section{Comparison of the VAD, blood flow, EDV, and PSV values in the observation group and unilateral VAH group}

The VAD, blood flow, EDV, and PSV values of the affected side of the observation group were significantly lower than those of the unilateral VAH group $(\mathrm{P}<0.05)$, while no significant differences were observed in the VAD, blood flow, EDV, VAD, and PSV values of the healthy side of the two groups $(\mathrm{P}>0.05$, Table 2$)$.

\section{Comparison of the RI and difference in RI between observation group and unilateral VAH group}

The RI of patients in the observation group was significantly higher than that of the unilateral VAH group, and the difference in RI was also significantly higher in the observation group than in the unilateral VAH group $(\mathrm{P}<0.05$, Table 3$)$.

\section{Assessment of the sensitivity and specificity of vertebral} artery intracranial stenosis by hemodynamic indicators

Figure 4 shows the results of receiver operating 


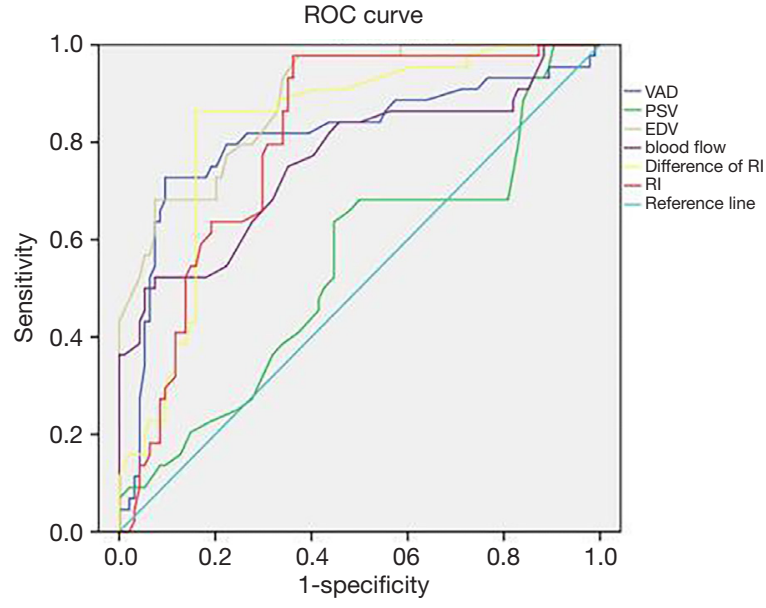

Figure 4 ROC curve analysis for evaluating the hemodynamic index of intracranial vertebral artery stenosis. ROC, receiver operating characteristic; VAD, vertebral artery diameter; PSV, peak systolic velocity; EDV, end diastolic velocity.

characteristic (ROC) curve analysis of hemodynamic indicators for assessing the presence of stenosis in the vertebral artery. Observation of the predicted limit of ROC curve movement was carried out. When the predicted limit of VAD was $2.084 \mathrm{~mm}$, the sensitivity and specificity were $86.43 \%$ and $49.45 \%$, respectively, and when the predicted limit of blood flow was $56.41 \mathrm{~mL} / \mathrm{min}$, the sensitivity and specificity were $88.67 \%$ and $47.42 \%$, respectively. When the predicted limit value of EDV was $8.47 \mathrm{~cm} / \mathrm{s}$, the sensitivity and specificity were $80.47 \%$ and $68.71 \%$ respectively. When the predicted limit value of the RI was 0.743 , the sensitivity and specificity were $82.71 \%$ and $84.66 \%$, respectively. When the predicted limit value of the difference in the RI was 0.149 , the sensitivity and specificity were $95.84 \%$ and $80.11 \%$, respectively.

\section{Discussion}

Ischemic cerebrovascular disease a nervous system disease in clinic. In recent years, with the changes in people's living habits and the deepening understanding of posterior circulation ischemia, the age of ischemic stroke onset has gradually become younger. Stenosis of the intracranial vertebral artery is one of the risk factors for posterior circulation ischemia, which can lead to cerebral infarction in the area of the brain supplied with blood by the vertebral artery $(7,8)$. Presently, early diagnosis and treatment of vascular stenotic lesions and ischemic lesions are highly valued in clinical research. Therefore, the appropriate choice of examination method is a key aspect in improving the detection rate of lesions.

Neck-brain integrated ultrasound is a new examination method developed on the basis of conventional ultrasound. It can dynamically display blood vessels in real time, effectively show intima-media in the blood vessels and the lesions of the intimal thickening, and measure the maximum flow rate, minimum flow rate, stenosis flow rate, RI, and other indexes $(9,10)$. In this study, the efficacy of neck-brain integrated ultrasound in the assessment of vascular stenosis was analyzed and compared with that of CTA and DSA. DSA has long been the "gold standard" for the clinical diagnosis of vascular stenosis lesions, because it can clearly show the entire blood vessels and assess whether stenosis is present, and it is also capable of showing the smoothness of the inner wall of the blood vessel (11). However, because DSA is an invasive procedure, the operation of which is quite complicated, it cannot be the preferred examination method. CTA, however, is non-invasive. Moreover, the neck blood vessel travels perpendicular to the CT scan cross-section, which makes it suitable for CTA examination. CTA imaging can also be read repeatedly with multi-angle and multi-directional scans, depicting clear vascular variations $(12,13)$. However, CTA examination involves $\mathrm{X}$-ray radiation, which poses a problem when there are high-density metals, such as dentures, in the patient's scanning area. This study showed that neck-brain integrated ultrasound has high specificity and sensitivity, and the diagnostic efficacy of moderate-to-severe stenosis and occlusive blood vessels was comparable to that of CTA and DSA, with no radiation damage. A number of foreign studies have indicated that ultrasound holds certain advantages in showing the degree and length of carotid artery stenosis (14).

As for the value of hemodynamic indexes of neckbrain integrated ultrasound for evaluating vertebral artery intracranial stenosis, hemodynamic markers have been shown to be the predictors of recurrent stroke in patients with intracranial stenosis (15). Therefore, current research in the neurosonology field is focusing on the early evaluation of hemodynamic changes in vertebral artery intracranial stenosis. Mohammaden et al. (16) highlighted that the smaller the inner diameter of the blood vessel, the greater the resistance to blood flow. Therefore, in the ultrasonic detection of hemodynamic parameters, the RI can be used as the main indicator of vascular resistance. This study showed that when the VAD was $\leq 2.5 \mathrm{~mm}$ and there 
was intracranial stenosis (the affected side of the observation group), the RI was significantly higher than in the unilateral VAH group. Furthermore, ROC curve analysis showed that the diagnostic efficiency of PSV was poor while EDV had a degree of sensitivity. However, the diagnostic sensitivity and specificity of RI were obviously better than those of EDV. Therefore, compared with PSV and EDV, the RI shows greater efficiency in evaluating intracranial segmental stenosis.

When unilateral VAH appears on one side of the blood vessel, the healthy vertebral artery may be partially compensated, the blood vessel diameter will increase, and the RI will decrease (17). The results of this study showed that the RI of the affected side in the observation group was significantly different from that of the healthy side $(\mathrm{P}<0.05)$. CTA and DSA were used as controls to evaluate the hemodynamic parameters of vascular ultrasound; when the difference of the intervertebral segment RI was 0.149 , the sensitivity and specificity of the diagnosis of vertebral artery intracranial stenosis were found to both exceed $80 \%$. Malota et al. (18) showed that the VAD became smaller in unilateral VAH, and the blood flow was proportional to the second power of the VAD. The blood flow was higher than that in the affected side of patients with vertebral artery stenosis, which was consistent with the findings of the above-mentioned studies. Additionally, ROC curve analysis in this study showed that the sensitivity of the intracranial vertebral artery stenosis based on blood flow was $88.67 \%$; however, the specificity was relatively poor.

Blood perfusion can be dynamically evaluated according to blood flow in real time, and intracranial vertebral artery can be diagnosed by transcranial color-coded Doppler sonography. When the VAD was $\leq 2.5 \mathrm{~mm}$ on one side, the blood perfusion was insufficient, so ultrasound imaging could not be used to accurately assess the intracranial vascular stenosis. CTA has a clear advantage in the assessment of anterior circulation perfusion, although it is inadequate for assessing posterior circulation perfusion $(19,20)$. Therefore, stenosis of the intracranial segment of the vertebral artery can be assessed sensitively according to the vascular ultrasound detection parameters, VAD, and the significant reduction in blood flow. However, the sample size in this study is small, analysis with larger sample size were needed in the further study.

In summary, as a non-invasive examination, neck-brain integrated ultrasound is of great value in the diagnosis of stenotic lesions of the internal cervical vertebral artery. Indexes including VAD, EDV, blood flow, RI, and the difference in RI can be used as important indicators to evaluate internal carotid stenosis of the vertebral artery, and may provide a reliable basis for the clinical diagnosis of posterior circulation ischemia.

\section{Acknowledgments}

Funding: This study was supported by the Key R\&D projects of the Autonomous Region (2018BEG03033).

\section{Footnote}

Reporting Checklist: The authors have completed the STROBE reporting checklist. Available at http://dx.doi. org/10.21037/atm-20-5713

Data Sharing Statement: Available at http://dx.doi. org/10.21037/atm-20-5713

Conflicts of Interest: All authors have completed the ICMJE uniform disclosure form (available at http://dx.doi. org/10.21037/atm-20-5713). The authors have no conflicts of interest to declare.

Ethical Statement: The authors are accountable for all aspects of the work in ensuring that questions related to the accuracy or integrity of any part of the work are appropriately investigated and resolved. This study was approved by General Hospital of Ningxia Medical University (2020-586). All procedures performed in this study involving human participants were in accordance with the Declaration of Helsinki (as revised in 2013). Because of the retrospective nature of the research, the requirement for informed consent was waived.

Open Access Statement: This is an Open Access article distributed in accordance with the Creative Commons Attribution-NonCommercial-NoDerivs 4.0 International License (CC BY-NC-ND 4.0), which permits the noncommercial replication and distribution of the article with the strict proviso that no changes or edits are made and the original work is properly cited (including links to both the formal publication through the relevant DOI and the license). See: https://creativecommons.org/licenses/by-nc-nd/4.0/.

\section{References}

1. Wang MY, Liu YS, Li K, et al. Protective effect of the 
microcatheter placed at the normal vertebral artery in intracranial stent-assisted angioplasty for vertebral artery stenosis: A case report. Medicine (Baltimore) 2017;96:e7569.

2. Saber H, Amiri A, Thrift AG, et al. Epidemiology of intracranial and extracranial large artery stenosis in a population-based study of stroke in the middle east. Neuroepidemiology 2017;48:188-92.

3. Rehman AA, Turner RC, Lucke-Wold BP, et al. Successful treatment of symptomatic intracranial carotid artery stenosis using a 24-mm long bare metal coronary stent. World Neurosurg 2017;102:693.e15-9.

4. Haquin A, Sigovan M, Si-Mohamed S, et al. Phasecontrast MRI evaluation of haemodynamic changes induces by a coeliac axis stenosis in the gastroduodenal artery. Br J Radiol 2017;90:20160802.

5. Gruber P, Braun C, Kahles T, et al. Percutaneous transluminal angioplasty using the novel drug-coated balloon catheter SeQuent Please NEO for the treatment of symptomatic intracranial severe stenosis: feasibility and safety study. J Neurointerv Surg 2019;11:719-22.

6. Kogue R, Maeda M, Umino M, et al. Evaluation of a highsignal lesion posterior to the intracranial vertebral artery using $3 \mathrm{D}$ balanced fast-field echo imaging. Neuroradiology 2019;61:1199-202.

7. Pelz JO, Weinreich A, Karlas T, et al. Evaluation of freehand B-mode and power-mode 3D ultrasound for visualisation and grading of internal carotid artery stenosis. PLoS One 2017;12:e0167500 .

8. Drazyk AM, Markus HS. Recent advances in the management of symptomatic vertebral artery stenosis. Curr Opin Neurol 2018;31:1-7.

9. Markus HS, Larsson SC, Kuker W, et al. Stenting for symptomatic vertebral artery stenosis: The vertebral artery ischaemia stenting trial. Neurology 2017;89:1229-36.

10. Lattanzi S, Brigo F, Di Napoli M, et al. Endovascular treatment of symptomatic vertebral artery stenosis: A systematic review and meta-analysis. J Neurol Sci 2018;391:48-53.

11. Siegler JE, Banwell B, Ichord RN. Teaching neuroimages: intracranial vertebral dissection in a 15 -year-old boy with sickle cell disease. Neurology 2016;87:e290-1.

12. Almadidy Z, Brunozzi D, Nelson J, et al. Intracranial venous sinus stenosis: hemodynamic assessment with twodimensional parametric parenchymal blood flow software on digital subtraction angiography. J Neurointerv Surg 2020;12:311-4.

13. Li Q, Hua Y, Jia L, et al. Abstract WMP40: accuracy of extracranial vertebral artery hemodynamic parameters in the evaluation of intracranial vertebral artery stenoocclusive disease. Stroke 2019;50:141-3.

14. Cheng Y, He W. Establishment of the intracranial hemodynamic model based on contrast medium and clinical applications. Medicine 2016;95:e5550.

15. Wabnitz AM, Derdeyn CP, Fiorella DJ, et al. Hemodynamic markers in the anterior circulation as predictors of recurrent stroke in patients with intracranial stenosis. Stroke 2018;50:A118020840.

16. Mohammaden MH, Rizwan HM, Denise B, et al. Role of resistivity index analysis in the prediction of hemodynamically significant venous sinus stenosis in patient with idiopathic intracranial hypertension. Neurosurgery 2020;86:631-6.

17. Yousef A, Hibbert B, Feder J, et al. A novel echocardiographic hemodynamic index for predicting outcome of aortic stenosis patients following transcatheter aortic valve replacement. PLoS One 2018;13:e0195641.

18. Malota Z, Glowacki J, Sadowski W, et al. Numerical analysis of the impact of flow rate, heart rate, vessel geometry, and degree of stenosis on coronary hemodynamic indices. BMC Cardiovasc Disord 2018;18:132.

19. Sigovan M, Bidet C, Bros S, et al. 3D black blood MR angiography of the carotid arteries. A simple sequence for plaque hemorrhage and stenosis evaluation. Magn Reson Imaging 2017;42:95-100.

20. Fatic N, Jaffer U, Ivana S, et al. Bilateral internal carotid artery occlusion, external carotid artery stenosis, and vertebral artery kinking: May it be asymptomatic? Ann Vasc Surg 2017;44:416.e5-8.

(English Language Editor: J. Reynolds)

Cite this article as: Wang J, Ma Q, Yang Z, Ma L. Feasibility of evaluating the stenosis of intracranial segment in the vertebral artery using neck-brain integrated ultrasound: a comparison with computed tomography angiography and digital subtraction angiography. Ann Transl Med 2020;8(18):1155. doi: 10.21037/atm-20-5713 